\title{
An improved method of constructing degradome library suitable for sequencing using Illumina platform
}

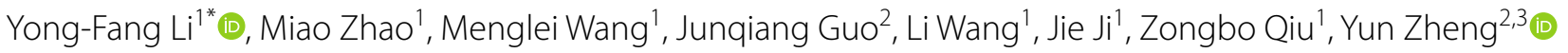 \\ and Ramanjulu Sunkar ${ }^{4^{*}}$ (D)
}

\begin{abstract}
Background: Post-transcriptional gene regulation is one of the critical layers of overall gene expression programs and microRNAs (miRNAs) play an indispensable role in this process by guiding cleavage on the messenger RNA targets. The transcriptome-wide cleavages on the target transcripts can be identified by analyzing the degradome or PARE or GMUCT libraries. However, high-throughput sequencing of PARE or degradome libraries using Illumina platform, a widely used platform, is not so straightforward. Moreover, the currently used degradome or PARE methods utilize Mmel restriction site in the $5^{\prime}$ RNA adapter and the resulting fragments are only 20-nt long, which often poses difficulty in distinguishing between the members of the same target gene family or distinguishing miRNA biogenesis intermediates from the primary miRNA transcripts belonging to the same miRNA family. Consequently, developing a method which can generate longer fragments from the PARE or degradome libraries which can also be sequenced easily using Illumina platform is ideal.

Results: In this protocol, $3^{\prime}$ end of the 5'RNA adaptor of TruSeq small RNA library is modified by introducing EcoP15I recognition site. Correspondingly, the double-strand DNA (dsDNA) adaptor sequence is also modified to suit with the ends generated by the restriction enzyme EcoP15I. These modifications allow amplification of the degradome library by primer pairs used for small RNA library preparation, thus amenable for sequencing using Illumina platform, like small RNA library.
\end{abstract}

Conclusions: Degradome library generated using this improved protocol can be sequenced easily using Illumina platform, and the resulting tag length is 27-nt, which is longer than the Mmel generated fragment (20-nt) that can facilitate better accuracy in validating target transcripts belonging to the same gene family or distinguishing miRNA biogenesis intermediates of the same miRNA family. Furthermore, this improved method allows pooling and sequencing degradome libraries and small RNA libraries simultaneously using Illumina platform.

Keywords: Cleavage, Degradome, Illumina sequencing, miRNA, Target gene

\section{Background}

The regulation of gene expression is controlled at multiple levels and the mRNA degradation/decay is one of the important determinants in this process. The mRNA

\footnotetext{
*Correspondence: li_yongfang@hotmail.com; ramanjulu.sunkar@okstate.edu ${ }^{1}$ College of Life Sciences, Henan Normal University, Xinxiang, Henan, People's Republic of China

${ }^{4}$ Department of Biochemistry and Molecular Biology, Oklahoma State University, Stillwater, OK 74078, USA

Full list of author information is available at the end of the article
}

degradation pathway is highly conserved in eukaryotes, and is controlled by exonucleases that can cause either $5^{\prime}$ to $3^{\prime}$ or $3^{\prime}$ to $5^{\prime}$ decay [1-4]. In addition, endonucleasedependent mRNA degradation which is guided by the small RNAs (miRNAs or siRNAs) emerged as yet another important conserved mRNA degradation pathways in higher eukaryotes [5, 6]. Plant miRNAs can cause degradation of the target mRNAs primarily by Argonaute (endonuclease)-mediated cleavage within the target site leaving a monophosphate at the $5^{\prime}$ end of the $3^{\prime}$ cleaved 
mRNA fragment $[7,8]$. Because plant miRNAs can target mRNAs that possesses perfect or near-perfect complementarity, their targets can be largely predicted using computational approaches $[9,10]$. However, false positive rate in such target predictions is high, therefore experimental validation is necessary. Modified 5' RACE (Rapid Amplification of cDNA Ends) is widely used technique to map in vivo cleavage sites induced by miRNA [11]. However, this approach is time consuming, labor-intensive and costly. To overcome these limitations, methods such as the PARE (parallel analysis of RNA ends) [12, 13], degradome [14] and GMUCT (genome-wide mapping of uncapped and cleaved transcripts) [15] that combine the $5^{\prime}$ RACE and high throughput sequencing of short reads have been developed. GMUCT technique generates variable length fragments for sequencing $[15,16]$, while, both PARE and degradome take advantage of MmeI digestion to generate consistent sized fragment (20-nt) (named as "tag" or "signature") derived from the $5^{\prime}$ end of $3^{\prime}$ cleaved product $[8,13,14]$. Detailed methodology of generating PARE or degradome libraries have been reported previously $[12,17]$. Moreover, by incorporating index into the library construction that allows multiplexing of degradome libraries for Illumina HiSeq sequencing, the PARE or degradome library construction has been further improved [18]. However, sequencing of the degradome or PARE library in an Illumina sequencer is complicated to some degree and not as straightforward as sequencing of other TruSeq libraries such as the small RNA library. This is due to the fact that $5^{\prime} \mathrm{RNA}$ adaptor length is varied between these two different libraries, i.e., the 5'RNA adaptor (RA5) of small RNA library is slightly longer than that of degradome or PARE library. Therefore, a specific PARE sequencing primer has to be used for sequencing. Regrettably, this sequencing primer is not compatible with the standard Illumina TruSeq sequencing primer, thus "SR_TubeStripHyb" manual must be used during cluster generation [18]. Another notable drawback with the currently-used degradome or PARE protocols is that these libraries yield reads or tags that are only 20-nt long, which poses difficulty in distinguishing between the members of the same target gene family.

Besides identifying miRNA targets, degradome or PARE libraries have the potential to reveal miRNA biogenesis $[8,13,19]$. The degradome tag analysis was instrumental in revealing the loop-first processing of MIR319 hairpins in plants [19]. However, surprisingly a significant number of degradome reads obtained from Arabidopsis [13], rice [8], Physcomitrella patens [19] and mouse [20] correspond to mature miRNAs suggesting that some of the miRNAs have been captured in degradome libraries. This could be due to adenylation of the mature miRNAs [21], or incomplete DCL1 cleavage (cleavage only at one arm of the hairpin of pri-miRNA), or loop-first cleavage during miRNA processing. This perplexity is largely due to similar size between mature miRNA reads and degradome reads. Therefore, generation of PARE or degradome tags longer than the length of canonical miRNA/miRNA* will not only improve accuracy in identifying miRNA targets but also in distinguishing between mature miRNA reads versus degradome reads. Additionally, the longer degradome read length can help in understanding the process of miRNA biogenesis. Although a restriction enzyme (EcoP15I) that can generate $\sim 27$-nt long reads was previously used in degradome libraries, the developed method was suitable for sequencing using Applied Biosystems SOLiD sequencing platform [19]. Given the advantages of Illumina sequencing, a detailed methodology that combines the use of EcoP15I and Illumina HiSeq sequencing platform is ideal. Indeed Zhai et al. [18] has modified the degradome protocol to suit to Illumina HiSeq platform but again MmeI restriction site was used in the RNA adapter. In this improved degradome or PARE protocol, longer read lengths are generated by using EcoP15I and the resulting libraries can be sequenced easily using Illumina sequencer (Fig. 1). Using this improved method, we have successfully constructed and sequenced degradome libraries from rice samples.

\section{Materials \\ Reagents}

TRIzol ${ }^{\circledR}$ reagent (ThermoFisher, Cat. No. 15596-026) Dynabeads $^{\circledR}$ mRNA purification kit (ThermoFisher, Cat. No. 610-06)

$3 \mathrm{M}$ sodium acetate $(\mathrm{NaOAc}), \mathrm{pH}=5.5$, RNase-free (ThermoFisher, Cat. No. AM9740)

Primers from TruSeq ${ }^{\circledR}$ small RNA sample prep kitSet A (Illumina, Cat. No. RS-200-0012)

T4 DNA ligase (ThermoFisher, Cat. No. 15224-017)

T4 RNA ligase (NEB, Cat. No. M0204S)

EcoP15I (NEB, Cat. No. R0646S)

Platinum $^{\circledR}$ Taq DNA polymerase high fidelity (ThermoFisher, Cat. No. 11304011)

20 bp DNA ladder (Takara, Cat. No. 3420A)

50 bp DNA ladder (Takara, Cat. No. 3421A)

DL 1000 DNA ladder (Takara, Cat. No. 3591A)

SuperScript $^{\mathrm{TM}}$ II reverse transcriptase (ThermoFisher, Cat. No. 18064)

RNaseOUT $^{\mathrm{TM}}$ recombinant ribonuclease inhibitor (ThermoFisher, Cat. No. 10777-019)

40\% Acrylamide/Bis19:1 40\% (w/v) solution (ThermoFisher, Cat. No. 9022)

$\mathrm{SYBR}^{\mathrm{TM}}$ gold nucleic acid gel stain (ThermoFisher, Cat. No. S11494) 


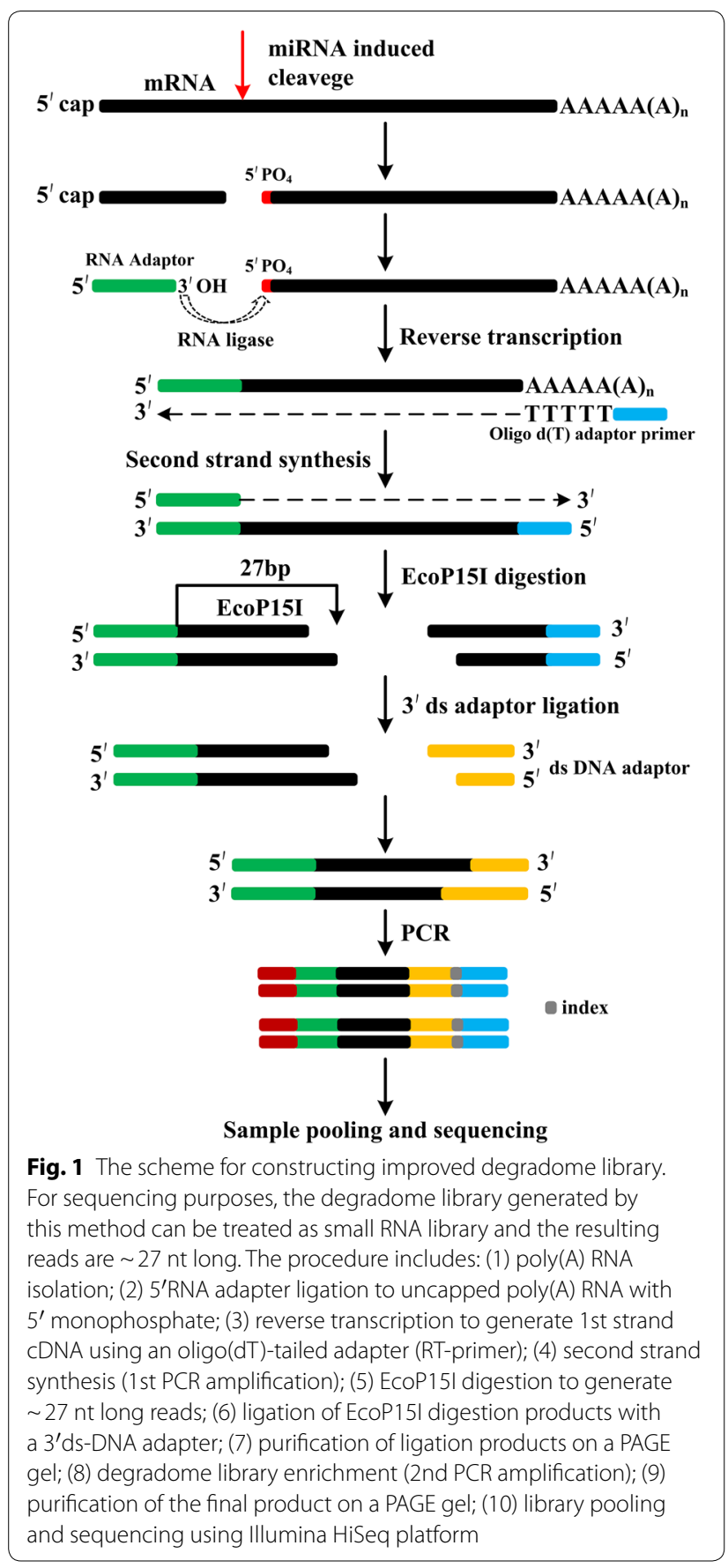

Ethidium bromide solution (Promega, Cat. No. H5041)

DEPC-treated water (ThermoFisher, Cat. No. AM9906)

Glycogen (ThermoFisher, Cat. No. 10814-010)

MinElute $^{\circledR}$ PCR purification kit (QIAGEN, Cat. No. 28004)
Corning_Costar_Spin-X_centrifuge tube filters (Sigma, Cat. No. CLS8162-24EA)

\section{Equipments}

Mini-protean tetra cell 4-gel vertical electrophoresis system (Biorad, Cat. No. 165-8001)

DynaMag $^{\mathrm{TM}}-2$ magnet (Thermo Fisher Scientific, Cat. No. 12321D)

NanoDrop One microvolume UV-vis spectrophotometer (Thermo Fisher Scientific, Cat. No. NDONE-W)

\section{Adapter and primer sequence}

5' RNA adapter: 5'-GUUCAGAGUUCUACAGUC CGACGAUCAGCAG-3' [this is the sequence of the $5^{\prime}$ RNA adaptor (RA5) of small RNA library with addition of AGCAG at $3^{\prime}$ end (bold and italic), which generate the recognition site of EcoP15I (underlined)].

RT-primer: 5'-CGAGCACAGAATTAATACGAC TTTTTTTTTTTTTTTTTTV-3'

$5^{\prime}$ adaptor primer: 5'-GTTCAGAGTTCTACA GTCCGAC-3'

$3^{\prime}$ adaptor primer: 5'-CGAGCACAGAATTAA TACGACT- $3^{\prime}$

dsDNA_top: 5'-NNTGGAATTCTCGGGTGCCA AGG-3' (PAGE purified)

dsDNA_bottom: 5'-CCTTGGCACCCGAGAATT CCA-3' (PAGE purified)

Final $5^{\prime}$ PCR primer: RP1 from TruSeq ${ }^{\circledR}$ Small RNA Sample Prep Kit

Final 3'PCR primer: indexed TruSeq 3' PCR primers, RPI1-12

\section{Protocol}

Total RNA sample preparation

The total RNA from plant tissues can be isolated using standard RNA isolation kits. We used TRIzol ${ }^{\circledR}$ Reagent for isolating total RNA from rice seedling [17]. Briefly, $0.2 \mathrm{mg}$ tissue was ground to fine powder and homogenized with $4 \mathrm{ml}$ of TRIzol ${ }^{\circledR}$ Reagent; after 5 min incubation at room temperature, $0.8 \mathrm{ml}$ chloroform was added and mixed well; following centrifugation, the upper aqueous phase was transferred to a new tube, and $2 \mathrm{ml}$ isopropanol was added to precipitate RNA; following centrifugation and $75 \%$ ethanol washing, RNA pellet was dissolved in DEPC $\mathrm{H}_{2} \mathrm{O}$. RNA quality and integrity are critical to the success of degradome libraries construction, which can be assessed by running on an 
agarose gel, using a Nanodrop spectrophotometer or Agilent's Bioanalyzer. RNA integrity can be checked by electrophoresis on a $1 \%$ agarose gel. Using Nanodrop, RNA concentration can be checked, and contaminations in RNA samples can be indicated by A260/280 and A260/230 ratios, which should be close to 1.8 and 2.0, respectively. If using a Bioanalyzer, RNA with high Integrity Number (RIN > 8.0) score is preferred (RIN score ranges from 1 to 10 and RIN 10 indicates highly intact RNA).

\section{Day 1}

\section{Poly(A) RNA purification}

We use the ThermoFisher Dynabeads mRNA Purification Kit to purify poly(A) RNA, but other mRNA purification kits should work as well. The initial amount of total RNA can be varied from 30 to $200 \mu$ g, and usage of higher quantities of initial total RNA will reduce the number of PCR cycles during enrichment of the final degradome library. We used $100 \mu \mathrm{g}$, and the volume of reagents and Dynabeads ${ }^{\mathrm{TM}}$ magnetic beads for poly(A) RNA purification were scaled based on the instructions (Thermofisher).

1. Initial RNA preparation: adjust the RNA volume to $135 \mu \mathrm{l}$ with DEPC-treated water. Denature the RNA at $65{ }^{\circ} \mathrm{C}$ for $2 \mathrm{~min}$ to disrupt secondary structures, and then immediately place on ice.

2. Prepare magnetic beads/binding buffer suspension:

a. Transfer $270 \mu \mathrm{l}$ of well resuspended Dynabeads ${ }^{\mathrm{TM}}$ magnetic beads to a $1.5 \mathrm{ml}$ eppendorf tube. Place the tube on a DynaMag ${ }^{\mathrm{TM}}-2$ magnet stand for $30 \mathrm{~s}$, or till all beads adhere to the tube wall, and then discard the supernatant.

b. Takeout the tube from the magnetic stand, and add $135 \mu$ l binding buffer to equilibrate the beads.

c. Put the tube back on the magnetic stand and discard the supernatant.

d. Takeout the tube from the stand, and add $135 \mu \mathrm{l}$ binding buffer to the beads.

3. $\operatorname{Poly}(\mathrm{A}) \mathrm{RNA}$ isolation:

a. Mix the total RNA from step 1 and the beads/ binding buffer suspension from step $2 \mathrm{~d}$.

b. Gently rotate the mixture using a roller for $5 \mathrm{~min}$ at room temperature to allow the poly(A) RNA annealing to the oligo $(\mathrm{dT})_{25}$ on the beads.

c. Place the tube on the magnetic stand till the solution become clear, discard the supernatant.

d. Remove the tube from the stand and wash the poly(A) RNA-bead complex twice with $270 \mu \mathrm{l}$ washing buffer B (carefully discard all traces of supernatant between each washing step).

e. Elute the poly $(\mathrm{A}) \mathrm{RNA}$ from the beads by adding $13 \mu \mathrm{l}$ of $10 \mathrm{mM}$ Tris- $\mathrm{HCl}$ ( $\mathrm{pH}$ 7.5). Keep the tube at $65^{\circ} \mathrm{C}$ for $2 \mathrm{~min}$ and then place immediately on the magnetic stand.

f. Transfer $12 \mu$ l eluted mRNA to a new RNase-free $1.5 \mathrm{ml}$ Eppendorf tube.

\section{Ligation of $5^{\prime} R N A$ adapter}

1. Add $1 \mu \mathrm{l} 5^{\prime}$ RNA adaptor $(100 \mu \mathrm{M})$ to $12 \mu \mathrm{l}$ mRNA, mix and incubate the tube at $65^{\circ} \mathrm{C}$ for 5 min to disrupt the secondary structure. Then place the tube on ice to chill ( $\sim 2 \mathrm{~min})$ and centrifuge briefly.

2. Add the following reagents to the poly(A) RNA/ adaptor mixture, mix thoroughly by pipetting up and down and centrifuge briefly. When multiple samples are handled, prepare master mix by multiplying the number of samples and plus $10 \%$ extra, add $7 \mu$ of the master mix to each poly(A) RNA/adaptor mixture.

\begin{tabular}{ll}
\hline Reagents & Volume $(\boldsymbol{\mu l})$ \\
\hline T4 RNA ligase buffer & 2 \\
$10 \mathrm{mM}^{\mathrm{l} T P}$ & 2 \\
RNaseOut $^{\mathrm{TM}}(40 \mathrm{U} / \mu \mathrm{l})$ & 1 \\
T4 RNA ligase $(5 \mathrm{U} / \mu \mathrm{l})$ & 2 \\
Total & 7 \\
\hline
\end{tabular}

3. Incubate the ligation reaction at $37{ }^{\circ} \mathrm{C}$ for $1 \mathrm{~h}$, and add $115 \mu$ of DEPC-treated water to the ligation reaction and proceed immediately to the next step.

\section{Purification of 5'RNA adapter ligated poly $(A)$ RNA}

Perform a 2nd round poly(A) RNA purification to remove the unincorporated $5^{\prime}$ RNA adapter and purify 5'RNA adaptor ligated poly(A) RNA. To do this, repeat the steps described in section "Poly(A) RNA purification" with the exception that final mRNA is eluted in $26 \mu \mathrm{l}$ of $10 \mathrm{mM}$ Tris-HCl ( $\mathrm{pH}$ 7.5). Transfer $25 \mu \mathrm{l}$ RNA adaptor ligated poly(A) RNA to a thin-walled PCR tube.

\section{First-stranded CDNA synthesis}

SuperScript ${ }^{\mathrm{TM}}$ II reverse transcriptase is used to synthesize the 1 st strand cDNA. SuperScript ${ }^{\mathrm{TM}}$ III and other reverse transcriptase can be used, and the components for the reverse transcription reaction should be adjusted accordingly. 
1. Add dNTPs and RT primer to the adaptor ligated poly(A) RNA and mix well.

\begin{tabular}{lc}
\hline Reagents & Volume $(\boldsymbol{\mu l})$ \\
\hline Adapter ligated mRNA & 25 \\
RT primer $(100 \mu \mathrm{M})$ & 2 \\
dNTP mix (10 $\mu$ M of each) & 2 \\
Total & 29 \\
\hline
\end{tabular}

2. Denature the mixture at $65{ }^{\circ} \mathrm{C}$ for $5 \mathrm{~min}$ to remove any RNA secondary structure, and then cool on ice.

3. Add 1st strand buffer, DTT and RNaseOUT, mix well and centrifuge briefly. Leave the tube at $42{ }^{\circ} \mathrm{C}$ for $2 \mathrm{~min}$

\begin{tabular}{lc}
\hline Reagents & Volume $(\boldsymbol{\mu l})$ \\
\hline $5 \times 1$ st strand Buffer & 10 \\
0.1 M DTT & 5 \\
RNase OUT & 2 \\
Total & 17 \\
\hline
\end{tabular}

4. Add $4 \mu \mathrm{l}$ SuperScript ${ }^{\mathrm{TM}}$ II reverse transcriptase, mix well and keep the tube at $42^{\circ} \mathrm{C}$ for $1 \mathrm{~h}$.

5. Incubate the reaction at $72{ }^{\circ} \mathrm{C}$ for $15 \mathrm{~min}$.

\section{First PCR amplification}

Use Platinum ${ }^{\circledR}$ Taq DNA Polymerase High Fidelity to prepare the 2nd strand cDNA.

1. Assemble the reaction in the following order.

\begin{tabular}{ll}
\hline Reagents & Volume $(\boldsymbol{\mu l})$ \\
\hline cDNA & 50 \\
$10 \times$ high fidelity PCR buffer & 10 \\
$50 \mathrm{mM} \mathrm{MgSO}_{4}$ & 4 \\
$\mathrm{dNTPs}(10 \mathrm{mM}$ of each) & 2 \\
$10 \mu \mathrm{M} \mathrm{5}$ ' adaptor primer & 2 \\
$10 \mu \mathrm{M} \mathrm{3} 3^{\prime}$ adaptor primer & 2 \\
Platinum ${ }^{\circledR}$ Taq DNA polymerase high fidelity & 0.4 \\
$\mathrm{H}_{2} \mathrm{O}$ & 29.6 \\
Total & 100 \\
\hline
\end{tabular}

2. Divide the PCR reaction into 3 thin-walled PCR tubes, and add $45 \mu \mathrm{l}$ each into two tubes, and the remaining $10 \mu \mathrm{l}$ into another tube (for experienced technicians, divide the PCR reaction into 2 tubes, $50 \mu \mathrm{l}$ each, and omit the following step 4).

3. PCR amplification. PCR reaction conditions: $94{ }^{\circ} \mathrm{C}$ for $2 \mathrm{~min}, 94{ }^{\circ} \mathrm{C}$ for $30 \mathrm{~s}, 58{ }^{\circ} \mathrm{C}$ for $30 \mathrm{~s}, 72{ }^{\circ} \mathrm{C}$ for 5 min, 7 cycles total, $72{ }^{\circ} \mathrm{C}$ for $5 \mathrm{~min}$, then hold at $4{ }^{\circ} \mathrm{C}$. For $10 \mu \mathrm{l}$ reaction, keep 15 PCR cycles.

4. Separate the $10 \mu \mathrm{l}$ PCR reaction on a $1 \%$ agarose gel. If smear ranging from 500 to $2500 \mathrm{bp}$ is visible (Additional file 1: Figure S1) which can indicate that the $5^{\prime}$ RNA adaptor ligation and 1st strand cDNA synthesis worked well. Then proceed to the next step.

PCR product purification using MinElute PCR purification kit PCR product is purified according to the procedure of MinElute PCR Purification using a microcentrifuge (QIAGEN).

1. Combine the PCR reaction $(90 \mu \mathrm{l})$ with 5 times volume of Buffer PB $(450 \mu \mathrm{l})$ containing $\mathrm{pH}$ indicator. If the color of the mixture is orange or violet, add $10 \mu \mathrm{l}$ of $3 \mathrm{M}$ sodium acetate, and mix well.

2. Place a MinElute column in a $2 \mathrm{ml}$ collection tube, and transfer the PCR/PB buffer mixture to the MinElute column and centrifuge at maximum speed for $1 \mathrm{~min}$ at room temperature.

3. Discard flow-through, and wash column by adding $750 \mu \mathrm{l}$ buffer PE and centrifuge at maximum speed for 1 min.

4. Discard flow-through and centrifuge the column for an additional $1 \mathrm{~min}$ at maximum speed.

5. Put the column into a new $1.5 \mathrm{ml}$ Eppendorf tube, add $12 \mu \mathrm{l}$ water at the center of the membrane, let the column stand for $1 \mathrm{~min}$, and then centrifuge for $1 \mathrm{~min}$, repeat this step again. Transfer $22 \mu$ lelution to a new tube.

\section{Digestion with EcoP15I}

Set up the digestion reaction in the following sequence:

\begin{tabular}{lc}
\hline Reagents & Volume $(\boldsymbol{\mu l})$ \\
\hline PCR product & 22 \\
$10 \times$ NEB buffer 3.1 & 3 \\
$10 \mathrm{mM}$ ATP & 3 \\
$10 \mathrm{U} / \mathrm{\mu l}$ ECOP15l & 2 \\
Total & 30 \\
\hline
\end{tabular}

1. Incubate the digestion at $37^{\circ} \mathrm{C}$ for $1-2 \mathrm{~h}$.

2. After digestion, inactivate EcoP15I at $65{ }^{\circ} \mathrm{C}$ for $20 \mathrm{~min}$, and then cool the digested mixture at room 
temperature (do not place the tube on ice). Proceed immediately to the next step.

\section{3'double-strand DNA (dsDNA) adapter ligation}

1. Prepare dsDNA adaptor. Mix equal amount of dsDNA_top and dsDNA_bottom oligos as shown in the table below. Mix well and centrifuge briefly and heat the mixture for $5 \mathrm{~min}$ at $100{ }^{\circ} \mathrm{C}$ and leave the tube at room temperature until it cools down. The dsDNA adapter should be prepared freshly each time, and this can be done during EcoP15I digestion.

\begin{tabular}{ll}
\hline Reagents & Volume $(\boldsymbol{\mu l})$ \\
\hline dsDNA_top $(100 \mu \mathrm{M})$ & 10 \\
dsDNA_bottom $(100 \mu \mathrm{M})$ & 10 \\
Total & 20 \\
\hline
\end{tabular}

2. Double strand DNA adaptor ligation. Set up the ligation mixture in the following order, mix well, centrifuge briefly and leave the ligation reaction at room temperature for $1 \mathrm{~h}$.

\begin{tabular}{lc}
\hline Reagents & Volume $(\boldsymbol{\mu l})$ \\
\hline EcoP15l digestion & 30 \\
$5 \times$ ligase buffer & 12 \\
dsDNA adaptor & 3 \\
T4 DNA ligase $(1 \mathrm{U} / \mu \mathrm{l})$ & 2 \\
$\mathrm{H}_{2} \mathrm{O}$ & 13 \\
Total & 60 \\
\hline
\end{tabular}

\section{PAGE purification of ligated dsDNA products (79 bp)}

1. Prepare $12 \%$ non-denaturing PAGE-TBE gel mixture in a $50 \mathrm{ml}$ conical vial in the following order. Then add $75 \mu \mathrm{l}$ of freshly prepared $10 \%$ ammonium persulfate (APS) and $15 \mu \mathrm{l}$ tetramethyl ethylene diamine (TEMED), mix well and cast a PAGE gel with $1.5 \mathrm{~mm}$ spacer. Prepare the PAGE gel during dsDNA adaptor ligation.

\begin{tabular}{ll}
\hline Reagents & Volume (for $\mathbf{1}$ gel) $(\mathbf{m l})$ \\
\hline $40 \%$ acrylamide stock $(\mathrm{ml})$ & 4.5 \\
$5 \times \mathrm{TBE}$ & 1.5 \\
$\mathrm{H}_{2} \mathrm{O}$ & 8.91 \\
Total & 15 \\
\hline
\end{tabular}

2. Prepare $0.5 \times$ TBE buffer.

3. Add $12 \mu \mathrm{l} 6 \times$ gel loading buffer to the ligation reaction, mix well and load samples in two wells. Leave 1 empty well between different samples if multiple samples are handled.

4. Load $20 \mathrm{bp}, 50 \mathrm{bp}$ DNA ladder at both sides of the samples.

5. Run gel in $0.5 \times$ TBE buffer till good separation (160 V, 1 h).

6. While running the gel, prepare $0.5 \mathrm{ml}$ tubes by puncturing one hole with a 21-gauge $(21 \mathrm{G})$ needle at the bottom, and place the tubes inside $2 \mathrm{ml}$ tubes.

7. Remove gel carefully and stain with $50 \mathrm{ml}$ of $1 \times$ SYBR gold in $0.5 \times$ TBE for 5 min by slowly shaking.

8. Visualize gel on a UV transilluminator. The ligated products should have a size of $79 \mathrm{bp}$ (79 bp $=5^{\prime}$ RNA adapter (31 bp) + EcoP15Idigested tag (27 bp) $+3^{\prime}$ dsDNA adapter (21 bp), but, the ligation band is not visible at this step, therefore, cut gel area corresponding to DNA ladder size between 70 and $90 \mathrm{bp}$ and put it into a $0.5 \mathrm{ml}$ tube with a hole (Fig. 2).

9. Centrifuge the gel pieces for 2 min at maximum speed; make sure all of gel pieces are in $2 \mathrm{ml}$ tube. Otherwise, puncture more holes in the $0.5 \mathrm{ml}$ tube and spin 1 min again.

10. Remove the $0.5 \mathrm{ml}$ tube and add $400 \mu \mathrm{H}_{2} \mathrm{O}$ to the $2 \mathrm{ml}$ tube.

11. Elute the ligation fragments overnight at $4{ }^{\circ} \mathrm{C}$ with gentle agitation.

\section{Days 2 and 3}

Concentrate the dsDNA adaptor ligated products by ethanol precipitation

1. Transfer the entire elution sample (suspension with gel) into a COSTAR Spin-X column centrifuge filter, spin at $12,000 \times g$ for $2 \mathrm{~min}$. Make sure all of the liquid spins out, and spin longer if necessary.

2. Measure the liquid volume, add $10 \%$ volume of $3 \mathrm{M}$ sodium acetate (NaOAc, pH 5.5), 2 volumes of $100 \%$ ethanol and $1 \mu \mathrm{l}$ glycogen. Mix well and leave the tube at $-80{ }^{\circ} \mathrm{C}$ for $2-3 \mathrm{~h}$.

3. Centrifuge at $12,000 \times g$ for $30 \mathrm{~min}$ at $4{ }^{\circ} \mathrm{C}$.

4. Discard supernatant, and wash the pellet with $70 \%$ ethanol, and centrifuge at $12,000 \times g$ for $5 \mathrm{~min}$ at $4{ }^{\circ} \mathrm{C}$.

5. Discard supernatant carefully and dry the pellet for 5 min at room temperature.

6. Dissolve the pellet in $40 \mu \mathrm{l} \mathrm{H}_{2} \mathrm{O}$. 


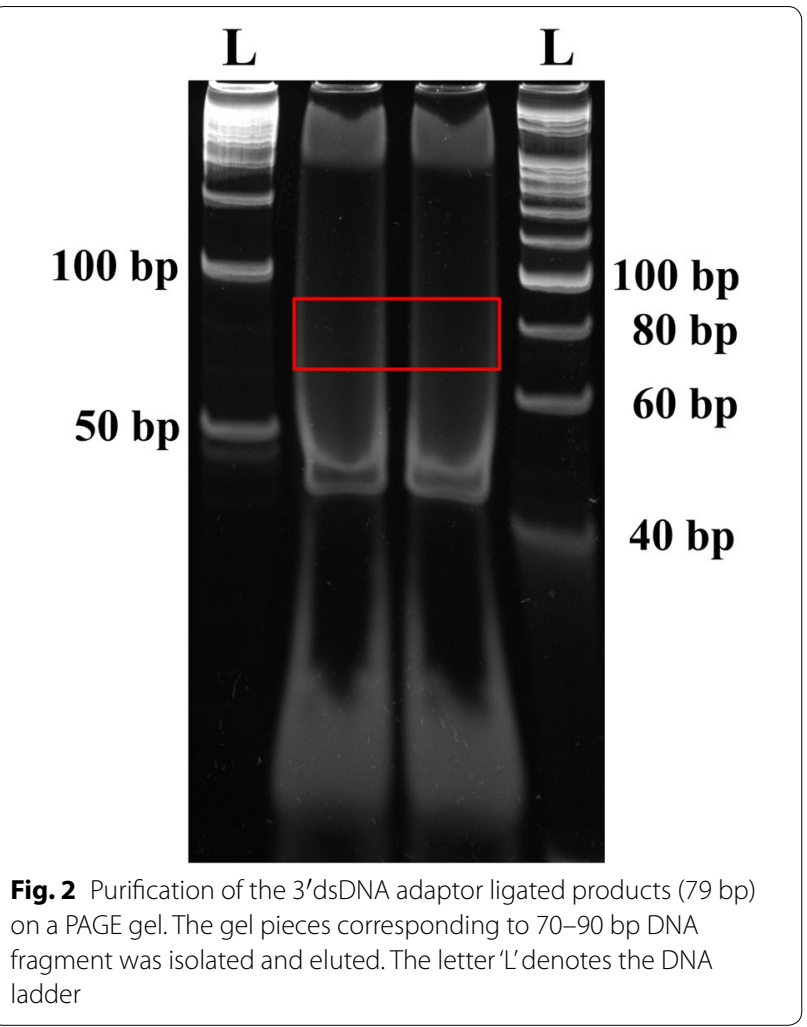

7. Transfer $39.8 \mu \mathrm{l}$ ligation product to a new thin wall PCR tube.

\section{PCR enrichment of degradome library}

1. Prepare PCR reaction in the following order.

\begin{tabular}{ll}
\hline Reagents & Volume $(\boldsymbol{\mu l})$ \\
\hline Ligation product & 39.8 \\
$10 \times$ high fidelity PCR buffer & 5 \\
$50 \mathrm{mM} \mathrm{MgSO}_{4}$ & 2 \\
dNTPs (10 mM each) & 1 \\
$10 \mu \mathrm{M} \mathrm{RP1}$ & 1 \\
$10 \mu \mathrm{M} 3^{\prime}$ index primer & 1 \\
Platinum ${ }^{\circledR}$ Taq DNA polymerase high fidelity & 0.2 \\
Total & 50 \\
\hline
\end{tabular}

2. Run PCR cycle: $94{ }^{\circ} \mathrm{C}$ for $2 \mathrm{~min}, 94^{\circ} \mathrm{C}$ for $30 \mathrm{~s}, 60{ }^{\circ} \mathrm{C}$ for $30 \mathrm{~s}, 72^{\circ} \mathrm{C}$ for $30 \mathrm{~s}, 11-15$ cycles, $72{ }^{\circ} \mathrm{C}$ for $5 \mathrm{~min}$, then hold at $4{ }^{\circ} \mathrm{C}$.

\section{PAGE purification of the final PCR products}

1. Prepare $8 \%$ non-denaturing PAGE gel (this can be done during the PCR amplification step). Prepare the gel mix in a $50 \mathrm{ml}$ conical vial in the following order. Then add $75 \mu \mathrm{l}$ freshly prepared 10\% APS and $15 \mu \mathrm{l}$ of TEMED. Mix well and cast a PAGE gel with $1.5 \mathrm{~mm}$ spacer.

\begin{tabular}{ll}
\hline Reagents & Volume (for $\mathbf{1}$ gel) $(\mathbf{m l})$ \\
\hline $40 \%$ acrylamide stock & 3 \\
$5 \times \mathrm{TBE}$ & 1.5 \\
$\mathrm{H}_{2} \mathrm{O}$ & 10.41 \\
Total & 14.91 \\
\hline
\end{tabular}

2. Add $10 \mu \mathrm{l}$ of $6 \times$ gel loading buffer to the final PCR reaction and load the PCR reaction into two wells. Meanwhile, load $50 \mathrm{bp}, 1 \mathrm{~kb}$ DNA ladder at left and right side of the samples.

3. Run gel in $0.5 \times$ TBE buffer till good separation $(120 \mathrm{~V}, 1 \mathrm{~h})$.

4. While running the gel, prepare $0.5 \mathrm{ml}$ tubes by puncturing one hole with a 21-gauge (21 G) needle at the bottom, and place it inside the $2 \mathrm{ml}$ tubes.

5. Remove gel carefully and stain the gel using $50 \mathrm{ml}$ of $0.5 \times$ TBE containing ethidium bromide for 5-10 min.

6. Visualize gel on transilluminator. The final PCR product should have a clear band near 150 bp DNA marker (Fig. 3a). Excise the PCR product band and put the gel pieces into the punctured $0.5 \mathrm{ml}$ tube.

7. Centrifuge the gel pieces for $2 \mathrm{~min}$ at maximum speed; make sure all of the gel pieces are in $2 \mathrm{ml}$ tube.

8. Discard the $0.5 \mathrm{ml}$ tube and add $400 \mu \mathrm{H}_{2} \mathrm{O}$ to the $2 \mathrm{ml}$ tube.

9. Elute the degradome library overnight at $4{ }^{\circ} \mathrm{C}$ with gentle agitation.

10. Repeat the same precipitation procedure as step "Concentrate the dsDNA adaptor ligated products by ethanol precipitation" with the exception that the final pellet is dissolved in $15 \mu \mathrm{l}$ nuclease-free water. 


\section{Quality assessment of degradome library and Illumina sequencing}

1. Determine the fragment size and purity of the degradome library using an Agilent Bioanalyzer High Sensitivity DNA chip. Optimal degradome library should have a tight fragment around $150 \mathrm{bp}$ (Fig. 3b).

2. Determine degradome library concentration by fluorometry (Qubit High Sensitivity Kit or Picogreen).

3. High throughput sequencing of degradome library. The degradome library prepared using this method can be treated as small RNA library for sequencing with single-end $50 \mathrm{nt}$ reads. Several degradome libraries can be pooled and multiplexed, like small RNA libraries.

\section{Results and discussion}

We aimed to improve the method for generating degradome libraries that can be easily sequenced using Illumina sequencer and can also yield longer read lengths. We generated degradome libraries of expected size of $150 \mathrm{bp}$ (Fig. 3). Using the small RNA library sequencing approach, we sequenced our degradome libraries that were of high quality (Additional file 2: Figure S2). The majority raw reads were 32-nt long, consisting of tag size of 27-nt, followed by 31- and 33-nt long raw reads, containing tags of 26-nt and 28-nt, respectively (Fig. 4). We further examined quality of raw reads, and $99 \%$ raw reads began with "AGCAG" (Fig. 5), which is derived from the nucleotides added to the $3^{\prime}$ end of $5^{\prime}$ RNA adaptor for generation of Ecop15I recognition site. The signature of "AGCAG" in raw reads, together with $95.75 \%$ raw reads of 31-33 nt long (Fig. 4), indicate the feasibility of usage of EcoP15I in degradome library generation. To identify plant miRNA targets, degradome data generated using this method can be analyzed using CleaveLand [22] or SeqTar [23] programs. The "AGCAG" signature need be trimmed from the raw reads prior to analyzing of the degradome reads.

Tags corresponding to mature miRNAs have been reported in Arabidopsis, Rice, moss and mouse [8, 13, 19, 20]. Using SeqTar pipeline [23], the degradome data from our previous study [8] and the present study was aligned to the precursors of the 22 evolutionary conserved miRNA families (miR156, miR159, miR160, miR162, miR164, miR166, miR167, miR168, miR169, miR171, miR172, miR319, miR390, miR393, miR394, miR395, miR396, miR397, miR398, miR399, miR408, and miR444). Sequence alignment of the 20-nt tags revealed that 48 precursors $(32 \%)$ had more than 5 reads exactly mapped to the beginning sites of miRNA-5p, and many tags could be mapped to multiple mature miRNAs belonging to the same miRNA family, although it is unknown whether these tags were derived from the adenylated miRNAs or incomplete DCL1 cleavage during miRNA biogenesis. Similar mapping of the rice degradome data generated in this study showed that only precursors of miR167h, miR168a and miR169i have tags more than 5 reads (30, 38 and 22 reads, respectively) mapped to the beginning sites of miRNA-5p. We further analyzed the origin of the 20-nt tags mapped to mature miRNAs

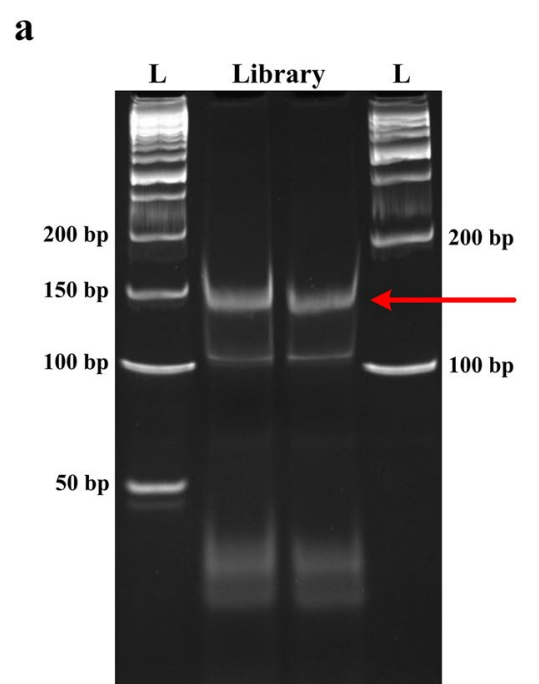

b

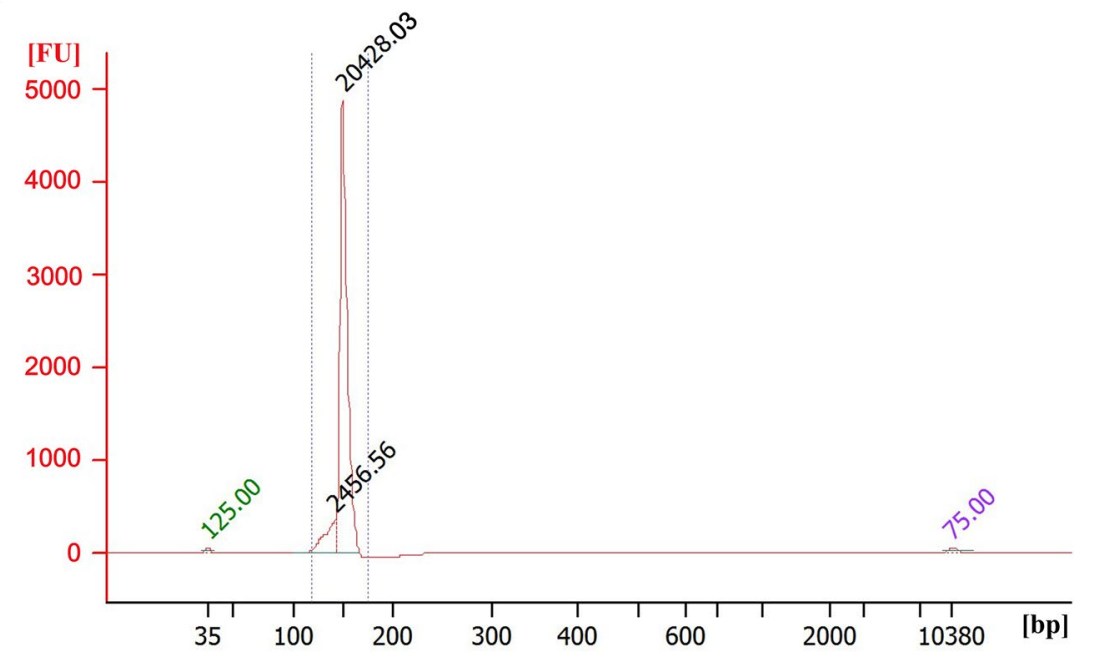

Fig. 3 Degradome library purification and quality assessment. a PAGE purification of the final PCR products ( $150 \mathrm{bp})$. The letter ' $\mathrm{L}$ ' denotes the DNA ladder. $\mathbf{b}$ Determination of fragment size profile of the degradome library using Agilent Bioanalyzer high sensitivity DNA chip. A clear peak at $\sim 150$ bp but no other peaks should be visible 


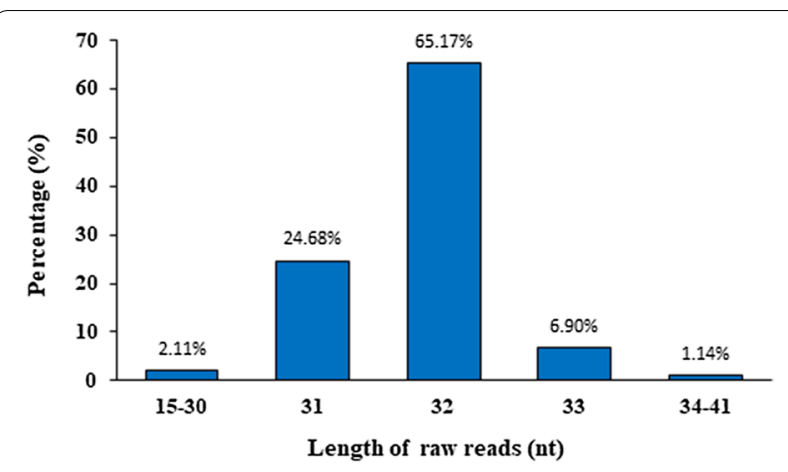

Fig. 4 Size distribution of the raw data generated from a rice degradome library

using the degradome data generated in this study, the outcome showed that the incomplete DCL1 cleavage on miRNA precursors is not common in rice. A 20-nt tag of TGCCTGGCTCCCTGTATGCC with 52 reads could be simultaneously mapped to the beginning site of miR164a, b, $d$ and $f$ (Fig. 6a, Additional file 3: Figure S3). If this tag was generated from DCL1 incomplete cleavage during miRNA biogenesis, the corresponding 27-nt tags from precursors of miR164a, b, $d$ and $f$ will be different from each other (Fig. 6a) and no such mapped tags were found in the 27-nt degradome data; if this tag was derived from miRNA164 adenylation, the corresponding 27-nt tags generated using this modified method cannot be mapped to the miR164 precursors. Indeed, we found 27-nt tags containing the 20-nt tag TGCCTGGCTCCCTGTATG $\mathrm{CC}$ which were largely derived from miR164 adenylation (Fig. 6b). Similarly, a 20-nt tag of TGAAGCTGC CAGCATGATCT with a frequency of 25 reads could be mapped to the beginning sites of miR167a, b, c, d, e, f, g, h, i and $j$ (Fig. 6c, Additional file 4: Figure S4). Using the present method, we found that this tag can be generated from not only miRNA167 adenylation, but also from the incomplete cleavage of rice miR167h precursor (Fig. 6d). These results clearly demonstrate that the $27-n t$ tags generated by the modified method can enhance the mapping accuracy of the reads.

Compared with the previous PARE protocol [18], the modifications included in this protocol are as follows: (1) altered 5'RNA adaptor: 5'RNA adaptor sequence in the previous protocol is $5^{\prime}$ GUUCAGAGUUCUACAGUCC GAC-3', which contains MmeI recognition site (underlined), and our modified 5'RNA adaptor sequence is

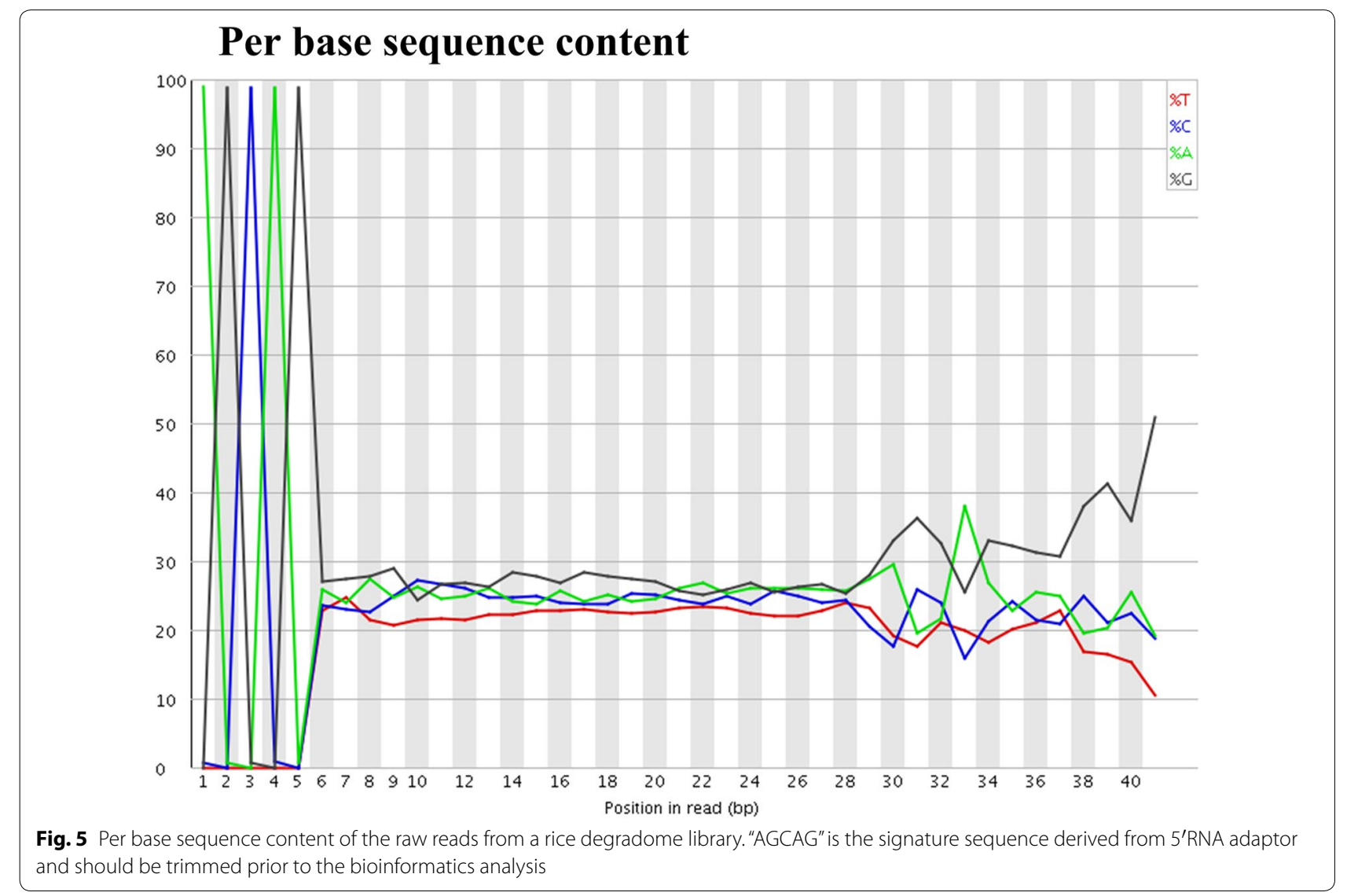




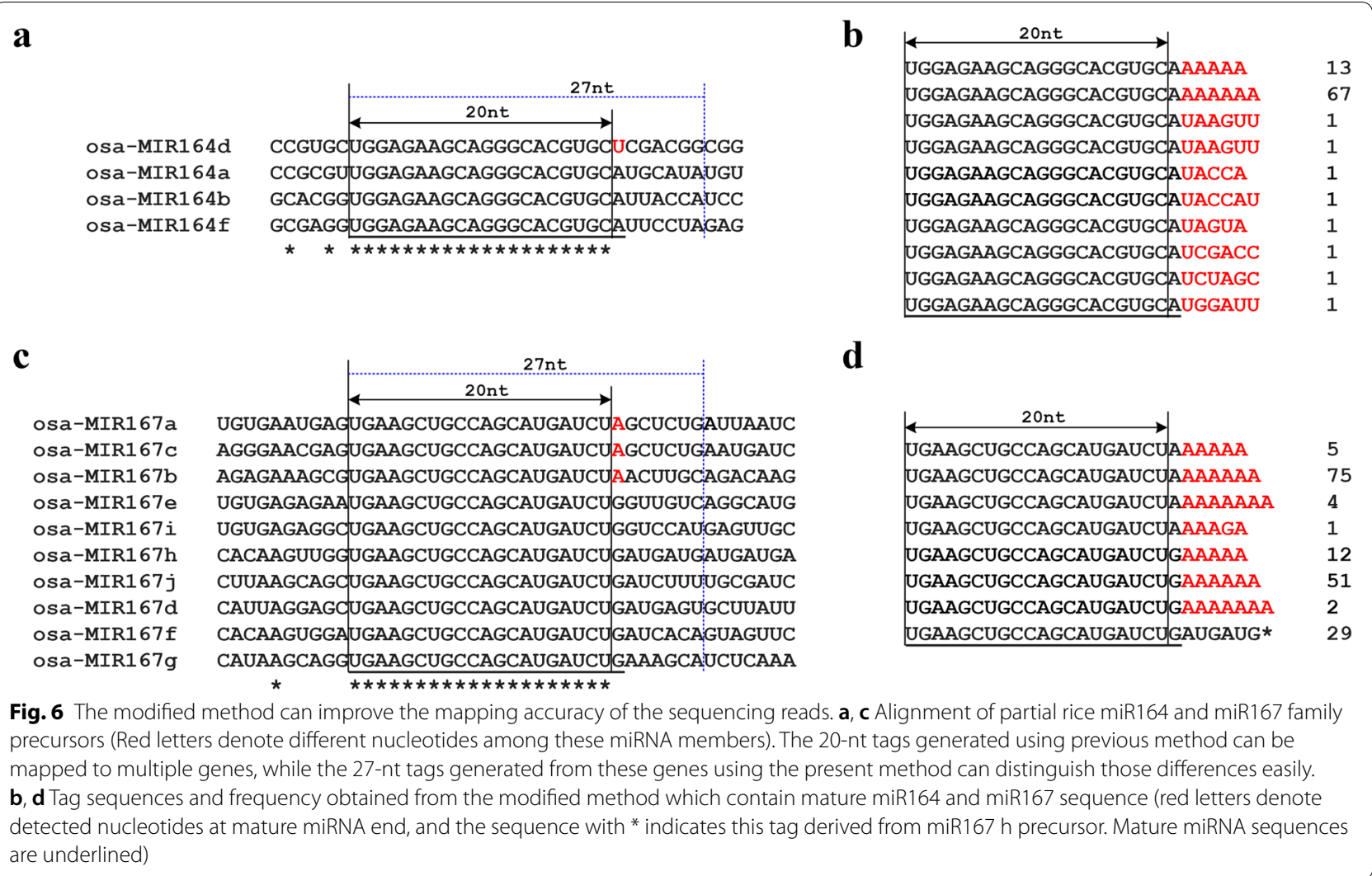

5'GUUCAGAGUUCUACAGUCCGACGAUCAGCAG $3^{\prime}$, which is longer (italics) than previous adaptor and contains the additional recognition site of EcoP15I (italics and underlined). (2) Agencourt $^{\circledR}$ AMPure $^{\circledR}$ XP (Beckman-Coulter) is convenient to purify 1st round PCR product when multiple PARE libraries are constructed, but we used MinElute ${ }^{\circledR}$ PCR purification kit (QIAGEN) to purify, which is quick and convenient for purifying PCR products when only a few samples are handled. Other brand PCR purification kits should work well too. (3) altered 3'dsDNA adapter: previously used top sequence: $5^{\prime}$ TGGAATTCTCGGGTGCCAAGG $3^{\prime}$, and bottom: 5' CCTTGGCACCCGAGAATTCCANN $3^{\prime}$; while the altered $3^{\prime}$ dsDNA adapter sequences are as follows (top) $5^{\prime}$ NNTGGAATTCTCGGGTGCCAAGG $3^{\prime}$, and (bottom) $5^{\prime}$ CCTTGGCACCCGAGAATTCCA $3^{\prime}$. (4) altered final $5^{\prime}$ PCR primer: previously used primer sequence is $5^{\prime}$ AATGATACGGCGACCACCGACAGG TTCAGAGTTCTACAGTCCGA $3^{\prime}$, however, RP1 from TruSeq ${ }^{\circledR}$ Small RNA Sample Prep Kit is used as final $5^{\prime}$ primer in this protocol. (5) previous PARE method generates degradome libraries of $128 \mathrm{bp}$ with tags of 20-nt, whereas this method generates the final libraries of 150 bp with tags of 26- to 28-nt, mainly 27-nt. (6) Illumina HiSeq sequencing of PARE library prepared by previous method must use PARE specific sequencing primer: 5' CCACCGACAGGTTCAGAGTTCTACAGT CCGAC $3^{\prime}$; The degradome library generated using this modified method can be sequenced in the same way as small RNA library, which is easier and more convenient. Therefore, degradome libraries generated using present method can even be pooled with small RNA libraries for sequencing. Even if the same index is used in both the libraries, i.e., degradome library and small RNA library, these libraries can still be pooled for sequencing, because degradome reads contain the "AGCAG" sequence signature that can be used to distinguish reads derived from degradome library rather than from small RNA library.

\section{Conclusions}

Here, we present a modified protocol for construction of degradome libraries, which can be used for studying degraded mRNAs with free $5^{\prime}$ monophosphates and poly(A) tail. Like previous methods [18], the entire protocol can be completed within 3 days. However, due to the introduction of EcoP15I recognition site at the $3^{\prime}$ end of 5'RNA adaptor of TruSeq small RNA library (RA5), the generated tag is $\sim 27-n t$ long. This facilitates a better accuracy in mapping the reads. The introduced 
modifications allow the libraries to be sequenced as Illumina TruSeq library. The degradome libraries can even be pooled with small RNA libraries for sequencing, which is convenient for analyzing both small RNAs and their targets simultaneously. The tags derived from miRNA precursor processing intermediate differ from tags of miRNA/miRNA* adenylation, therefore, this method can also be used for gaining insights into miRNA biogenesis.

\section{Supplementary information}

Supplementary information accompanies this paper at https://doi. org/10.1186/s13007-019-0524-7.

Additional file 1: Figure S1. Visualization of the 1st PCR product. PCR product was separated using $1 \%$ agarose gel. The letter ' $L$ ' denotes the DNA ladder

Additional file 2: Figure S2. Quality scores across all bases of a rice degradome library raw data.

Additional file 3: Figure S3. Signature abundance throughout the length of rice miR164 precursors. The 20-nt tags generated by previous method [8] were plotted on to rice miR164 precursors; miRNA precursor sequences were extended 50-nt at $5^{\prime}$ and $3^{\prime}$ end, respectively. Arrows indicate the beginning site of miR164.

Additional file 4: Figure S4. Signature abundance throughout the length of rice miR167 precursors. The 20 -nt tags generated by previous method [8] were plotted on to the rice miR167 precursors; miRNA precursor sequences were extended 50-nt at $5^{\prime}$ and $3^{\prime}$ end, respectively. Arrows indicate the beginning site of miR167.

\section{Acknowledgements}

We thank OEBIOTECH (Shanghai, China) for high-throughput sequencing of the generated rice degradome library.

\section{Authors' contributions}

$Y L$ and RS conceived the idea and designed the protocol described. MZ, MW and LW isolated total RNA and performed mRNA purification, YL, MZ and MW constructed degradome library. YL, JG, JJ, ZQ and $Y Z$ analyzed the data. $Y L$ and RS wrote the manuscript. All authors read and approved the final manuscript.

\section{Funding}

This work was supported by grants from National Natural Science Foundation of China [NO. 31771703 \& 31601241$]$ and a startup fund from Henan Normal University to $\mathrm{YL}$.

\section{Availability of data and materials}

The degradome data obtained in this study was deposited at the National Center for Biotechnology Information Gene Expression Omnibus (NCBI, GEO, http://www.ncbi.nlm.gov.geo/) under accession number GSE138545.

\section{Ethics approval and consent to participate}

Not applicable.

\section{Consent for publication}

Not applicable.

\section{Competing interests}

The authors declare that they have no competing interests.

\section{Author details}

${ }^{1}$ College of Life Sciences, Henan Normal University, Xinxiang, Henan, People's Republic of China. ${ }^{2}$ Faculty of Information Engineering and Automation, Kunming University of Science and Technology, Kunming 650500, Yunnan, China. ${ }^{3}$ Yunnan Key Laboratory of Primate Biomedical Research, Institute of Primate Translational Medicine, Kunming University of Science and Technology,
Kunming 650500, China. ${ }^{4}$ Department of Biochemistry and Molecular Biology, Oklahoma State University, Stillwater, OK 74078, USA.

Received: 2 July 2019 Accepted: 9 November 2019

Published online: 18 November 2019

\section{References}

1. Meyer S, Temme C, Wahle E. Messenger RNA turnover in eukaryotes: pathways and enzymes. Crit Rev Biochem Mol Biol. 2004;39(4):197-216.

2. Garneau NL, Jeffrey W, Wilusz CJ. The highways and byways of mRNA decay. Nat Rev Mol Cell Biol. 2007:8(2):113-26.

3. Ghildiyal M, Zamore P. Small silencing RNAs: an expanding universe. Nat Rev Genet. 2009;10(2):94-108.

4. Yu X, Willmann MR, Anderson SJ, Gregory BD. Genome-wide mapping of uncapped and cleaved transcripts reveals a role for the nuclear mRNA cap-binding complex in cotranslational RNA decay in Arabidopsis. Plant Cell. 2016;28(10):2385-97.

5. Voinnet O. Origin, biogenesis, and activity of plant microRNAs. Cell. 2009;136(4):669-87.

6. Jones-Rhoades MW, Bartel DP, Bartel B. MicroRNAs and their regulatory roles in plants. Annu Rev Plant Biol. 2006;57:19-53.

7. Axtell MJ. Classification and comparison of small RNAs from plants. Annu Rev Plant Biol. 2013;64(1):137-59.

8. Li YF, Zheng Y, Addo-Quaye C, Zhang L, Saini A, Jagadeeswaran G, Axtell MJ, Zhang W, Sunkar R. Transcriptome-wide identification of microRNA targets in rice. Plant J. 2010;62(5):742-59.

9. Jones-Rhoades MW, Bartel DP. Computational identification of plant microRNAs and their targets, including a stress-induced miRNA. Mol Cell. 2004;14(6):787-99.

10. Rhoades MW, Reinhart BJ, Lim LP, Burge CB, Bartel B, Bartel DP. Prediction of plant microRNA targets. Cell. 2002;110(4):513-20.

11. Llave C, Xie Z, Kasschau KD, Carrington JC. Cleavage of scarecrowlike mRNA targets directed by a class of Arabidopsis miRNA. Science. 2002;297(5589):2053-6.

12. German MA, Luo S, Schroth G, Meyers BC, Green PJ. Construction of parallel analysis of RNA ends (PARE) libraries for the study of cleaved miRNA targets and the RNA degradome. Nat Protoc. 2009;4(3):356-62.

13. German MA, Pillay M, Jeong DH, Hetawal A, Luo S, Janardhanan P, Kannan V, Rymarquis LA, Kan N, German R. Global identification of microRNAtarget RNA pairs by parallel analysis of RNA ends. Nat Biotechnol. 2008;26(8):941-6

14. Addo-Quaye C, Eshoo TW, Bartel DP, Axtell MJ. Endogenous siRNA and miRNA targets identified by sequencing of the Arabidopsis degradome. Curr Biol. 2008;18(10):758-62.

15. Gregory B, O'Malley R, Lister R, Urich M, Tonti-Filippini J, Chen H, Millar A, Ecker J. A link between RNA metabolism and silencing affecting Arabidopsis development. Dev Cell. 2008;14(6):854-66.

16. Willmann MR, Berkowitz ND, Gregory BD. Improved genome-wide mapping of uncapped and cleaved transcripts in eukaryotes-GMUCT 2.0. Methods. 2014;67(1):64-73.

17. Li YF, Sunkar R. Global identification of small RNA targets in plants by sequencing sliced ends of messenger RNAs. Methods Mol Biol. 2013:956:119-29.

18. Zhai J, Arikit S, Simon SA, Kingham BF, Meyers BC. Rapid construction of parallel analysis of RNA end (PARE) libraries for Illumina sequencing. Methods. 2014;67(1):84-90

19. Addo-Quaye C, Snyder JA, Park YB, Li YF, Sunkar R, Axtell MJ. Sliced microRNA targets and precise loop-first processing of MIR319 hairpins revealed by analysis of the Physcomitrella patens degradome. RNA. 2009;15(12):2112-21.

20. Bracken CP, Szubert JM, Mercer TR, Dinger ME, Thomson DW, Mattick JS, Michael MZ, Goodall GJ. Global analysis of the mammalian RNA degradome reveals widespread miRNA-dependent and miRNA-independent endonucleolytic cleavage. Nucleic Acids Res. 2010;39(13):5658-68.

21. Lu S, Sun YH, Chiang VL. Adenylation of plant miRNAs. Nucleic Acids Res. 2009;37(6):1878-85.

22. Addo-Quaye C, Miller W, Axtell MJ. CleaveLand: a pipeline for using degradome data to find cleaved small RNA targets. Bioinformatics. 2009:25(1):130-1. 
23. Zheng Y, Li YF, Sunkar R, Zhang W. SeqTar: an effective method for identifying microRNA guided cleavage sites from degradome of polyadenylated transcripts in plants. Nucleic Acids Res. 2012;40(4):e28.

\section{Publisher's Note}

Springer Nature remains neutral with regard to jurisdictional claims in published maps and institutional affiliations.
Ready to submit your research? Choose BMC and benefit from:

- fast, convenient online submission

- thorough peer review by experienced researchers in your field

- rapid publication on acceptance

- support for research data, including large and complex data types

- gold Open Access which fosters wider collaboration and increased citations

- maximum visibility for your research: over $100 \mathrm{M}$ website views per year

At BMC, research is always in progress.

Learn more biomedcentral.com/submissions 